Series A

\author{
I. MATHEMATICA
}

505

\title{
EXISTENCE OF BOUNDED DIRICHLET FINITE BIHARMONIC FUNCTIONS
}

BY

MITSURU NAKAI and LEO SARIO

H E L S I N K I 1972

S U O M A L A N E N T I E D A K A T E M A

doi:10.5186/aasfm.1972.505 
Copyright (C) 1972 by

Academia Scientiarum Fennica

ISBN $951-41-0032-8$

Communicated 5 November 1971 by Pentti Laasonen 


\section{EXISTENCE OF BOUNDED DIRICHLET FINITE BIHARMONIC FUNCTIONS}

In 1950 Virtanen [6] proved that if the Laplace equation $\Delta u=0$ on an open Riemann surface $R$ possesses a nonconstant Dirichlet finite solution then it also possesses a nonconstant bounded Dirichlet finite solution. Here the Dirichlet integral $D_{R}(u)$ of $u$ is $\int_{R}|\operatorname{grad} u(z)|^{2} d x d y$. Let $P(z) d x d y$ be a nonnegative Hölder-continuous second-order differential on $R$. In 1952 Ozawa [4] remarked that the phenomenon just mentioned is valid for the equation $(A-P) u=0$ if the Dirichlet integral is replaced by the energy integral $E_{R}(u)=D_{R}(u)+\int_{R} u(z)^{2} P(z) d x d y$. Recently Nakai [2] proved that even the genuine phenomenon is also valid for the equation $(\Delta-P) u=0$.

We assume that $\lambda(z) d x d y$ is a strictly positive $C^{2}$ second-order differential on $R$. We consider the equation

$$
\Delta\left(\lambda(z)^{-1} \Delta u(z)\right)=0
$$

or more generally

$$
(\Delta-Q(z))\left(\lambda(z)^{-1}(\Delta-P(z)) u(z)\right)=0
$$

where $Q(z) d x d y$ is similar to $P(z) d x d y$. The solutions of $\Delta u=0$ or $(\Delta-P) u=0$ are also solutions of (1) or (2) and therefore it may be interesting to see whether the Virtanen phenomenon continues to be valid for the equations (1) and (2). The main result of this paper is that the Virtanen phenomenon may not necessarily occur even for the simpler equation (1), i.e. we will prove the following

Main theorem. There exists a parabolic plane region $R$ and a strictly positive $C^{\infty}$ function $\lambda$ on $R$ such that the equation $\Delta\left(\lambda^{-1} \Delta u\right)=0$ has nonconstant Dirichlet finite solutions on $R$ but none of them are bounded on $R$.

The solutions of $\Delta\left(\lambda^{-1} \Delta u\right)=0$ are called biharmonic on $(R, \lambda)$ and their relations to analytic functions are well-known. The equation $\Delta\left(\lambda^{-1} \Delta u\right)=0$ is equivalent to the family of Poisson equations

The work was done under the 1971 summer employment by Grant DA-AROD-31-124-71-G20, UCLA. 


$$
\Delta u(z)=-\lambda(z) h(z)
$$

for harmonic functions $h$ on $R$. Therefore (1) admits a nonconstant Dirichlet finite solution if and only if (3) does for some $h$. For this reason we will first give a complete condition for (3), or a slightly more general equation, to possess a Dirichlet finite solution. After this preparation we will give an example proving our main theorem.

\section{Existence theorems}

1. Let $\mu(z) d x d y$ be a Hölder-continuous second-order differential on an open Riemann surface $R$ such that $\mu(z) \geq 0$ and $\mu(z) d x d y \neq 0$. We denote by $H_{\mu}(R)$ the class of $h \in H(R), h \neq 0$, such that the Poisson equation

$$
\Delta u(z)=-\mu(z) h(z)
$$

possesses a Dirichlet finite solution on $R$. If $R^{\prime} \subset R$, then $H_{\mu}(R) \subset H_{\mu}\left(R^{\prime}\right)$. We first give complete conditions for an $h \in H(R)$ to belong to $H_{\mu}(R)$. For this purpose let $\{\Omega\}$ be an exhaustion of $R$ by regular subregions $\Omega$. We denote by $G_{\Omega}(z, \zeta)$ the Green kernel on $\Omega$. If $R \notin O_{G}$ (cf. e.g. Sario-Nakai [5]), then the Green kernel on $R$ will be denoted by $G_{R}(z, \zeta)$. or simply by $G(z, \zeta)$. We also consider the class $D(R)$ of functions $\varphi$ such that the Dirichlet integrals $D_{R}(\varphi)$ can be defined and are finite. We then have

Theorem 1. For an $h \in H(R), h \neq 0$, the following three conditions are equivalent by pairs:

$(\alpha) h \in H_{\mu}(R)$;

$(\beta) \sup _{\varphi \in C_{0} D(R)}\left|\int_{R} h(z) \varphi(z) \mu(z) d x d y\right|^{2} / D_{R}(\varphi)<\infty ;$

$(\gamma) \sup _{\Omega} \int_{\Omega \times \Omega} G_{\Omega}(z, \zeta) h(z) h(\xi) \mu(z) \mu(\xi) d x d y d \xi d \eta<\infty$ for one and hence for every exhaustion $\{\Omega\}$ of $R$.

For simplicity we use the notation $(h, \varphi)_{\mu}$ for the integral

$$
\int_{R} h(z) \varphi(z) \mu(z) d x d y
$$

and $G_{\Omega}(h, h)_{\mu}$ for the integral in $(\gamma)$. Clearly $(., .)_{\mu}$ is a semi-inner product and the same is true for $G_{\Omega}(., .)_{\mu}$ because of the energy principle for Green potentials (cf. e.g. Constantinescu-Cornea [1]).

Suppose first that $h \in H_{\mu}(R)$ and $u$ is a solution of (4) with $u \in D(R)$. Since the Stokes formula yields $(h, \varphi)_{\mu}=(\Delta u, \varphi)=-D_{R}(u, \varphi)$ for $\varphi \in C_{0}^{\infty}(R)$ and for $\varphi \in C_{0} D(R)$ by approximation, the Schwarz inequality 
implies $(\beta)$. Conversely suppose that $(\beta)$ is valid. Let $\mathcal{F}$ be the Hilbert space obtained by the completion of $C_{0}^{\infty}(R)$ with respect to the inner product $D_{R}(.,$.$) . By (\beta), \varphi \rightarrow(h, \varphi)_{\mu}$ is a bounded linear functional on $C_{0}^{\infty}(R)$ considered as a subspace of $\mathcal{F} \subset D$, and therefore it can be extended to 7 as a bounded linear functional $\varphi \rightarrow l(\varphi)$. By the Riesz theorem there exists a $u \in \mathcal{J}$ such that $l(\varphi)=D_{R}(u, \varphi)$ for every $\varphi \in \mathcal{F}$. In particular $(h, \varphi)_{\mu}=D_{R}(u, \varphi)$ for every $\varphi \in C_{0}^{\infty}(R)$. Let

$$
\bar{u}_{\Omega}(z)=\frac{1}{2 \pi} \int_{\Omega} G(z, \zeta) h(\zeta) \mu(\zeta) d \xi d \eta .
$$

Since $\Delta \bar{u}=-\mu h, D_{\Omega}(u-\bar{u}, \varphi)=0$ for every $\varphi \in C_{0}^{\infty}(\Omega)$. By the Weyl lemma, $u-\bar{u} \in H(\Omega)$ and a fortiori $u$ is a solution of (4) on $\Omega$. Here the arbitrariness of $\Omega$ implies that $u$ is a solution of (4) on $R$, and $u \in D(R)$. Therefore $h \in H_{\mu}(R)$, i.e. the equivalence of $(\alpha)$ and $(\beta)$ is proved.

Once more suppose that $h \in H_{\mu}(R)$ and $u$ is a solution of (4) with $u \in D(R)$. Let $\{\Omega\}$ be the exhaustion of $R$ consisting of every regular subregion $\Omega$ of $R$. By a simple application of the Green formula we

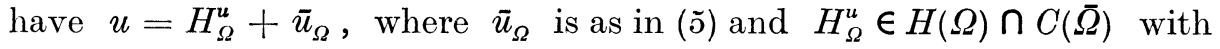
$H_{\Omega}^{u} \mid \partial \Omega=u$. Therefore $D_{\Omega}\left(\bar{u}_{\Omega}\right) \leq D_{\Omega}(u) \leq D_{R}(u)$. Observe that

$$
D_{\Omega}\left(\bar{u}_{\Omega}\right)=G_{\Omega}(h, h)_{\mu},
$$

which is known as the Evans relation; its proof is an easy consequence of the Green formula. Hence we conclude that $(\gamma)$ follows. Conversely suppose that $(\gamma)$ is valid for one exhaustion $\{\Omega\}$ of $R$. Because of (6), $\left\{\bar{u}_{\Omega}\right\}$ has a weak limit $u$ in e.g. $\mathcal{F}$, which can be seen, as in the proof of the equivalence of $(\alpha)$ and $(\beta)$, to be a solution of (4) on $R$ with $u \in D(R)$. Therefore $(\alpha)$ and $(\gamma)$ are equivalent.

2. Let $S$ be a Riemann surface and $\alpha$ be a part of the ideal boundary such that $(S, \alpha)$ is a bordered surface with analytic boundary $\alpha \neq \varnothing$, compact or noncompact. We denote by $\hat{S}$ the double of $S$ about $\alpha$. Let $j$ be the involution of $\hat{S}$, i.e. a mapping of $\hat{S}$ such that $z$ and $j(z)$ are symmetric about $\alpha$. An $h \in H(\widehat{S})$ is said to be antisymmetric if $h(j(z))=$ $-h(z)$ on $\hat{S}$. Let $\mu(z) d x d y$ be as in no. 1 and $\zeta=j(z)$. If $\mu(\zeta) d \xi d \eta=$ $\mu(z) d x d y$, then we say that $\mu(z) d x d y$ or $\mu$ is symmetric. As a specialization of Theorem 1 we have

Theorem 2. Let $h$ be an antisymmetric harmonic function on $\hat{S}$ and $\mu(z) d x d y$ be a nonnegative symmetric Hölder-continuous second-order differential $\equiv 0$ on $\hat{S}$. Suppose that $h \geq 0$ on the support of $\mu(z) d x d y$ in $S$. Then $h \in H_{\mu}(\hat{S})$ if and only if 


$$
\int_{S \times S} G_{S}(z, \zeta) h(z) h(\zeta) \mu(z) \mu(\zeta) d x d y d \xi d \eta<\infty .
$$

The significance of this theorem manifests itself when $\hat{S} \in O_{G}$. Obsərve that $S$ always carries the Green kernel since it has an analytic border $\alpha$. For the proof of the theorem, let $\{\Omega\}$ be an exhaustion of $\hat{S}$ such that $j(\Omega)=\Omega$, and set $\omega=\Omega \cap S$. The key relation is

$$
G_{\Omega}(z, \zeta)-G_{\Omega}(j(z), \zeta)=G_{\omega}(z, \zeta)
$$

for $z$ and $\zeta$ in $\omega$. In view of (8), the antisymmetry of $h$, and the symmetry of $\mu$, we deduce

$$
G_{\Omega}(h, h)_{\mu}=2 G_{(\omega)}(h, h)_{\mu} .
$$

By Theorem 1, $h \in H_{\mu}(S)$ is equivalent to the boundedness of $\left\{G_{\Omega}(h, h)_{\mu}\right\}_{\Omega}$, and in turn to the boundedness of $\left\{G_{\omega}(h, h)_{\mu}\right\}_{\Omega}$. Since $h(z) h(\zeta) \geq 0$ on the support of $\mu(z) \mu(\zeta) d x d y d \xi d \eta$ in $S \times S$, the boundedness of $\left\{G_{\omega}(h, h)_{\mu}\right\}_{\Omega}$ is equivalent to (7). This completes the proof of Theorem 2.

3. By definition we excluded the constant function 0 from $H_{\mu}(R)$ and hence $H_{\mu}(R)$ cannot form a vector space. However we can at least say that

$$
\left(\mathbf{R} H_{\mu}(R)+\mathbf{R} H_{\mu}(R)\right)-\{0\} \subset H_{\mu}(R),
$$

i.e. $H_{\mu}(R) \cup\{0\}$ is a vector space. The proof is obvious. The following relation will be used repeatedly:

$$
\bigcap_{j=1}^{n} H_{\mu_{j}}(R) \subset{\underset{j=1}{\sum_{j=1}^{n}}(R)}(R) .
$$

This is also a direct consequence of the definition of $H_{\mu}(R)$.

\section{An example}

4. We will construct an example to prove our main theorem. We denote by $\mathbf{C}$ the finite complex plane and by $\mathbf{P}$ the right half-plane. The basic surface we are going to consider is the doubly-punctured plane:

$$
\mathbf{C}^{\prime}=\mathbf{C}-\{-3,3\} \text {. }
$$

This is a parabolic plane region. Set $\mathbf{P}^{\prime}=\mathbf{P}-\{3\}$. Observe that

$$
\mathbf{C}=\hat{\mathbf{P}}, \mathbf{C}^{\prime}=\hat{\mathbf{P}}^{\prime},
$$

with respect to $\partial \mathbf{P}$, the imaginary axis. The $R$ in the main theorem 
will be $\mathbf{C}^{\prime}$. In order to construct $\lambda$ on $\mathbf{C}^{\prime}$ with the property described in the main theorem, we will consider six auxiliary functions (differentials) $\mu_{1}, \ldots, \mu_{6}$. We first consider a positive rotation-free $C^{\infty}$ function $\mu_{1}$ on $\mathrm{C}$ such that $\mu_{1} \mid\{|z| \leq 4\}=1$ and

$$
\mu_{1}(z)=(1+|z|)^{-(3+\alpha)}(0<\alpha<1)
$$

on $\{|z| \geq 5\}$ in $\mathbf{C}$ and also in $\mathbf{C}^{\prime}$. The reason for this choice is clarified by Nakai [3], though it is not needed for the present purpose. The elementary functions

$$
c(z)=(z+\bar{z}) / 2, s(z)=(z-\bar{z}) / 2 i
$$

will play important roles. First we assert:

Lemma 1. The functions $c$ and $s$ belong to $H_{\mu_{1}}(\mathbf{C}) \subset H_{\mu_{1}}\left(\mathbf{C}^{\prime}\right)$.

Since the proof is the same, we will only prove $c \in H_{\mu_{1}}(\mathbf{C})$. Take an arbitrary $\varphi \in C_{0}^{\infty}(R)$ and expand it into its Fourier series:

$$
\varphi\left(r e^{i \Theta}\right)=\sum_{n=0}^{\infty}\left(a_{n}(r) \cos n \Theta+b_{n}(r) \sin n \Theta\right) .
$$

In the double integral $(c, \varphi)_{\mu_{1}}$ we first integrate with respect to $\Theta$ :

$$
(c, \varphi)_{\mu_{1}}=O\left(\int_{0}^{\infty} a_{1}(r) r^{2}(1+r)^{-(3+\alpha)} d r\right) .
$$

By the Schwarz inequality, $\mid(c, \varphi)_{u_{1}}{ }^{2} \leq K \int_{0}^{\infty} a_{1}(r)^{2} r^{-1} d r$, where

$$
K=O\left(\int_{0}^{\infty} r^{5}(1+r)^{-2(3+\alpha)} d r\right)<\infty .
$$

On the other hand, $D_{\mathbf{C}}(\varphi) \geq \pi \int_{0}^{\infty} a_{1}(r)^{2} r^{-1} d r$, and a fortiori

$$
\left|(c, \varphi)_{\mu_{1}}\right|^{2} \leq K D_{\mathbf{C}}(\varphi) \text {. }
$$

Thus by Theorem 1 we conclude that $c \in H_{\mu_{1}}(\mathbf{C})$.

5. Next take a rotation-free nonnegative $C^{\infty}$ function $\varphi$ on $\mathbf{C}$ with its support in the disk $|z|<\frac{1}{2}$ such that $\varphi \equiv 1$ in a neighborhood of $z=0$. Fix a decreasing sequence $\left\{\varepsilon_{n}\right\}_{1}^{\infty}$ of positive numbers such that $\sum_{1}^{\infty} \varepsilon_{n}<\infty$, and choose an increasing sequence $\left\{\eta_{n}\right\}_{1}^{\infty}$ of positive numbers such that $\eta_{1}>6, \varepsilon_{n}\left(\eta_{n}-1\right)>1$, and $\left(\eta_{n}+1\right)^{2}<\eta_{n+1}+1$ for every $n=1,2, \ldots$ We consider a function

$$
\mu_{2}(z)=\sum_{n=1}^{\infty} \varepsilon_{n} \varphi\left(z-i \eta_{n}\right)
$$

where $i$ is the imaginary unit. Then we have 
Lemma 2. The function $c$ belongs to $H_{\mu_{2}}(\mathbf{C}) \subset H_{\mu_{2}}\left(\mathbf{C}^{\prime}\right)$ but $s$ does not belong to $H_{\mu_{2}}\left(\mathbf{C}^{\prime}\right)$.

By Theorem 2 we only have to show that $G_{\mathbf{P}}(c, c)_{\mu_{2}}<\infty$. By the energy principle of Green potentials,

$$
\left(G_{\mathbf{P}}(c, c)_{\mu_{2}}\right)^{1 / 2} \leq \sum_{n=1}^{\infty}\left(G_{\mathbf{P}}\left(c \varepsilon_{n} \varphi\left(\cdot-i \eta_{n}\right), c \varepsilon_{n} \varphi\left(\cdot-i \eta_{n}\right)\right)_{1}\right)^{1 / 2} .
$$

The $n^{\text {th }}$ term of the sum on the right is given by

$$
\varepsilon_{n}^{2} \underset{\left(\left|z-i \eta_{n}\right|<1\right) \cap \mathbf{P} \times\left(\left|\zeta-i \eta_{n}\right|<1\right) \cap \mathbf{P}}{ } \log \left|\frac{z+\bar{\zeta}}{z-\zeta}\right| \cdot x \xi \varphi\left(z-i \eta_{n}\right) \varphi\left(\zeta-i \eta_{n}\right) d x d y d \xi d \eta
$$

and by changing the variables from $z$ and $\zeta$ to $z-i \eta_{n}$ and $\zeta-i \eta_{n}$, we obtain for the above integral the form $\varepsilon_{n}^{2} a^{2}$ with

$$
\mathrm{a}=\left(\int_{(|z|<1) \cap \mathbf{P} \times(|\xi|<1) \cap \mathbf{P}} \log \left|\frac{z+\bar{\zeta}}{z-\zeta}\right| \cdot x \xi \varphi(z) \varphi(\zeta) d x d y d \xi d \eta\right)^{1 / 2}<\infty .
$$

Therefore $\quad\left(G_{\mathbf{P}}(c, c)_{\mu_{2}}\right)^{1 / 2} \leq a \sum_{n=1}^{\infty} \varepsilon_{n}<\infty$, and $c \in H_{\mu_{2}}(\mathbf{C})$.

To show that $s \notin H_{\mu_{2}}\left(\mathbf{C}^{\prime}\right)$, we denote by $\mathbf{Q}$ the upper half-plane and observe that $\mathbf{C}^{\prime}=\hat{\mathbf{Q}}$ with respect to $\partial \mathbf{Q}-\{-3,3\}$. Since $s>0$ on $\mathbf{Q}$, we have

$$
G_{\mathbf{Q}}(s, s)_{\mu} \geq \sum_{n=1}^{\infty} G_{\mathbf{Q}}\left(s \varepsilon_{n} \varphi\left(\cdot-i \eta_{n}\right), s \varepsilon_{n} \varphi\left(\cdot-i \eta_{n}\right)\right)_{1} .
$$

The $n^{\text {th }}$ term of the sum on the right is given by

$$
\begin{aligned}
& \varepsilon_{n}^{2} \int_{\left(\left|z-i \eta_{n}\right|<\frac{1}{2}\right) \times\left(\left|\zeta-i \eta_{n}\right|<\frac{1}{2}\right)} \log \left|\frac{z-\bar{\zeta}}{z-\zeta}\right| \cdot y \eta \varphi\left(z-i \eta_{n}\right) \varphi\left(\zeta-i \eta_{n}\right) d x d y d \xi d \eta \\
= & \varepsilon_{n}^{2} \int_{\left(|z|<\frac{1}{2}\right) \times\left(|\xi|<\frac{1}{2}\right)} \log \left|\frac{z-\bar{\zeta}+2 i \eta_{n}}{z-\zeta}\right| \cdot\left(y+\eta_{n}\right)\left(\eta+\eta_{n}\right) \varphi(z) \varphi(\zeta) d x d y d \xi d \eta \\
\geq & \varepsilon_{n}^{2} \int_{\left(|z|<\frac{1}{2}\right) \times\left(|\xi|<\frac{1}{2}\right)} \log \frac{1}{|z-\zeta|} \cdot\left(\eta_{n}-1\right)\left(\eta_{n}-1\right) \varphi(z) \varphi(\zeta) d x d y d \xi d \eta \\
\geq & \int_{\left(|z|<\frac{1}{2}\right) \times\left(|\xi|<\frac{1}{2}\right)} \log \frac{1}{|z-\zeta|} \cdot \varphi(z) \varphi(\zeta) d x d y d \xi d \eta \equiv a>0 .
\end{aligned}
$$

Therefore $G_{\mathbf{Q}}(s, s)_{\mu_{2}} \geq \sum_{n=1}^{\infty} a=\infty, \quad$ and by Theorem 2, $s \notin H_{\mu_{2}}(\hat{\mathbf{Q}})=$ $H_{\mu_{2}}\left(\mathbf{C}^{\prime}\right)$. 
6. Next take a $C^{\infty}$, rotation-free about 3 , nonnegative function $\mu_{3}(z)$ on $\mathbf{C}^{\prime}$ vanishing identically on $|z-3| \geq \frac{1}{2}$ in $\mathbf{C}^{\prime}$ such that

$$
\mu_{3}(z)=(|z-3| \log |z-3|)^{-2}
$$

on $0<|z-3| \leq 1 / 4$ in $\mathbf{C}^{\prime}$. Let $\mu_{4}(z)$ be the function on $\mathbf{C}^{\prime}$ which is symmetric to $\mu_{3}(z)$ with respect to the imaginary axis.

To define the fifth function $\mu_{5}(z)$, we take a strictly decreasing sequence $\left\{r_{n}\right\}_{0}^{\infty}$ such that $r_{0}=1$ and $\lim _{n} r_{n}=0$. Let $w_{n} \in H\left(\mathbf{P}-\left\{|z-3| \leq r_{n}\right\}\right)$ $\cap C(\overline{\mathbf{P}})$ such that $w_{n} \mid\left\{|z-3| \leq r_{n}\right\}=1$ and $w_{n} \mid \partial \mathbf{P}=0$. Since $\left\{w_{n}\right\}_{1}^{\infty}$ converges to 0 uniformly on each compact subset of $\mathbf{P}^{\prime}$, we can choose $\left\{r_{n}\right\}_{0}^{\infty}$ so that $D_{\mathbf{P}}\left(w_{n}\right)<4^{-n}$. We then regularize $w_{n}$ so as to obtain a $C^{\infty}$, rotation-free about 3, superharmonic function $\omega_{n}$ such that $\omega_{n}=w_{n}$ on $\left\{|z-3|>r_{n-1}\right\} \cup\left\{|z-3|<r_{n+1}\right\}$ in $\overline{\mathbf{P}}$ with

$$
D_{\mathbf{P}}\left(\omega_{n}\right) \leq 4^{-n} \quad(n=1,2, \ldots) \text {. }
$$

Then define

$$
\mu_{5}(z)=-\left(\sum_{n=1}^{\infty} \Delta \omega_{n}(z)\right) / c(z) \geq 0 .
$$

At each point $z \in \mathbf{P}^{\prime}$, the sum is a finite sum. Outside of $|z-3| \leq 1$ in $\mathbf{P}^{\prime}, \mu_{5}(z)=0$. Thus we can consider $\mu_{5} C^{\infty}$ on $\mathbf{C}^{\prime}$. Let $\mu_{6}$ be the function on $\mathbf{C}^{\prime}$ which is symmetric to $\mu_{5}$ with respect to the imaginary axis.

Let $\omega$ be an antisymmetric function on $\mathbf{C}^{\prime}$ with respect to $\partial \mathbf{P}$ such that

$$
\omega(z)=\sum_{n=1}^{\infty} \omega_{n}(z)
$$

on $\mathbf{P}^{\prime}$. The sequence is convergent because of $(16)$ and $D_{\mathbf{C}^{\prime}}(\omega)<\infty$. Actually $\omega(z)$ is a positive $C^{\infty}$ superharmonic function on $\mathbf{P}^{\prime}$ such that

$$
\lim _{z \in \mathbf{P}^{\prime}, z \rightarrow 3} \omega(z)=\infty \text {. }
$$

It is readily seen that $\Delta$ and the sum can be interchanged in the calculation of $\Delta \omega$ in (18) and therefore by (17) we obtain

$$
\Delta \omega(z)=-\left(\mu_{5}(z)+\mu_{6}(z)\right) c(z)
$$

on $\mathbf{C}^{\prime}$. In particular $c \in H_{\mu_{5}+\mu_{6}}\left(\mathbf{C}^{\prime}\right)$. We now have

Lemma 3. The function $c$ belongs to $H_{\mu_{3}+\mu_{4}+\mu_{5}+\mu_{6}}\left(\mathbf{C}^{\prime}\right)$.

By (11) we only have to show that $c \in H_{\mu_{3}+\mu_{4}}\left({ }^{\prime}\right) \mathbf{C}$. However $G_{\mathbf{P}}(c, c)_{\mu_{3} \times \mu_{4}}=G_{\mathbf{P}}(c, c)_{\mu_{3}} \leq \int_{\mathbf{P} \times \mathbf{P}} G_{\mathbf{P}}(z, \zeta) \mu_{3}(z) \mu_{3}(\zeta) d x d y d \xi d \eta$. In order to show that $G_{\mathbf{P}}(c, c)_{\mu_{3}+\mu_{4}}<\infty$, it is sufficient by Theorem 2 to prove 
$a=\sup _{\varphi \in C_{0}^{\infty}(\mathbf{P})} \mid(1, \varphi)_{\mu_{3}}{ }^{2} / D_{\mathbf{P}}(\varphi)<\infty$. As in the proof of Lemma 1, we deduce

$$
(1, \varphi)_{\mu_{3}}=O\left(\int_{0}^{1 / 2} a_{0}(r)(r \log r)^{-2} r d r\right) .
$$

By the Schwarz inequality

$$
\begin{aligned}
\left|(1, \varphi)_{\mu_{3}}\right|^{2} & \leq O\left(\int_{0}^{\infty} a_{0}(r) r^{-1} d r\right)\left(\int_{0}^{1 / 2} r^{-1}(\log r)^{-4} d r\right) \\
& =O\left(\int_{0}^{\infty} a_{0}(r) r^{-1} d r\right) .
\end{aligned}
$$

On the other hand, $D_{\mathbf{P}}(\varphi) \geq 2 \pi \int_{0}^{\infty} a_{0}(r)^{2} r^{-1} d r$, and a fortiori $a<\infty$.

7. Finally we define a strictly positive $C^{\infty}$ function $\lambda$ on $\mathbf{C}^{\prime}$ by

$$
\lambda(z)=\sum_{n=1}^{6} \mu_{n}(z) .
$$

Lemma 4. Let $U=\{|z-3|<1 / 4\}$ and $U^{\prime}=U-\{3\}$. If $h \in H_{\lambda}\left(U^{\prime}\right)$, then $h \in H(U)$.

Let $h_{0}$ be the regular part $h$ in $V=\{|z-3|<1 / 8\}$, i.e. $h_{0} \in H(V)$. Since $G_{V}\left(h_{0}, h_{0}\right)_{u_{n}} \leq k^{2} G_{V}(1,1)_{u_{n}}$ with $k=\sup _{V}\left|h_{0}\right|$, we can conclude that $h_{0} \in H_{\mu_{n}}\left(V^{\prime}\right)$ with $V^{\prime}=V-\{3\}$ if $\sup _{\varphi} \in C_{0}^{\infty}(V):\left.(1, \varphi)_{u_{n}}\right|^{2} / D_{V}(\varphi)<\infty$. For $n=2,4$, and 6 this is trivial. For $n=1$ and 3 it can be shown as in the proof of Lemma 3. Observe that $(1, \varphi)_{u_{s}}=-\left(\Delta(\omega, \varphi / c)_{1}=D_{V}(\omega, \varphi / c)\right.$. By the Schwarz inequality, $\mid(1, \varphi)_{\mu_{0}}{ }^{2} \leq D_{V}(\omega)\left(O\left(D_{V} \cdot(\varphi)+\left(1, \varphi^{2}\right)_{1}\right)\right)$. Clearly $\left(1, \varphi^{2}\right)_{1} \leq O\left(D_{V}(\varphi)\right)$ and thus $h_{0} \in H_{\mu_{n}}(V)$ for $n=1, \ldots, 6$. Therefore $h_{0} \in H_{\lambda}(V)$. For this reason we may assume that $h$ lacks the regular part in the proof of the above lemma. Then, in terms of $z-3=r e^{i \theta}$, $h$ takes the form

$$
h\left(r e^{i \Theta}\right)=a \log r+\sum_{n=1}^{\infty} r^{-n}\left(a_{n} \cos n \Theta+b_{n} \sin n \Theta\right) .
$$

We wish to show that $h \equiv 0$. First suppose that $a_{n}^{2}+b_{n}^{2} \neq 0$ for some $n$. Let $\varrho_{t, t^{\prime}}(r)=\left(r-t^{\prime}\right)(t-r)$ for $t^{\prime} \leq r \leq t$ and 0 for $r>t$ or $t^{\prime}>r \geq 0$. Set $\varphi_{t, t^{\prime}}\left(r e^{i \theta}\right)=\varrho_{t, t^{\prime}}(r)\left(a_{n} \cos n \Theta+b_{n} \sin n \Theta\right)$. By Theorem 1 there exists a constant $K$ such that

$$
\left.\mid\left(h, \varphi_{t, t^{\prime}}\right)_{\lambda}\right]^{2}=O\left(\int_{t^{\prime}}^{t} r^{-n} \varrho_{t, t^{\prime}}(r) \lambda(r) r d r\right)^{2} \leq K D_{U}\left(\varphi_{t, t^{\prime}}\right) .
$$


Here the fact that $\lambda\left(r e^{i \theta}\right)=\lambda(r)$ is important. By letting $t^{\prime} \rightarrow 0$, we obtain

$$
\left(\int_{0}^{t} r^{-n+2}(t-r) \lambda(r) d r\right)^{2} \leq O\left(D_{U}\left(\varphi_{t}\right)\right)
$$

where $\varphi_{t}\left(r e^{i \Theta}\right)=r(t-r)\left(a_{n} \cos n \Theta+b_{n} \sin n \Theta\right)$. Since $\mu_{3}<\lambda$,

$$
\left(\int_{0}^{t} r^{-n+2}(t-r)(r \log r)^{-2} d r\right)^{2} \leq O\left(D_{U}\left(\varphi_{t}\right)\right) .
$$

The first integral is $O\left(t^{-n+2}\right)$ and $D_{U}\left(\varphi_{t}\right)=O\left(t^{4}\right)$. Hence $O\left(t^{-n+2}\right) \leq O\left(t^{2}\right)$ or $O\left(t^{-n}\right) \leq O(1)$, a contradiction since $t^{-n} \rightarrow \infty(t \rightarrow 0)$.

Next assume $a \neq 0$ and take $\varphi\left(r e^{i \theta}\right)=a \log (\max (r, t))$. Chouse the rotation-free (with respect to 3 ) $\varphi_{m} \in C_{0}^{\infty}\left(U^{\prime}\right)$ such that $\sup _{U}\left|\varphi_{m}-\varphi\right|+$ $D_{U}\left(\varphi_{m}-\varphi\right)^{1 / 2} \rightarrow 0$. Since

$$
\left|\left(h, \varphi_{m}\right)_{\lambda}\right|^{2}=O\left(\int_{0}^{1 / 4} \log r \cdot \varphi_{m}(r) \cdot \lambda(r) r d r\right)^{2} \leq K D_{U}\left(\varphi_{m}\right)
$$

and $\mu_{3}<\lambda$, we deduce on letting $m \rightarrow \infty$

$$
O\left(\int_{t}^{1 / 4}(\log r)^{2} \cdot(r \log r)^{-2} r d r\right)^{2} \leq O\left(\int_{t}^{1 / 4} r^{-2} \cdot r d r\right)
$$

or $O(\log t)^{2} \leq O(\log t)$. This means that $O\left({ }_{i} \log t\right) \leq O(1)$ as $t \rightarrow \infty$, a contradiction.

Lemma 5. The class $H_{\lambda}\left(\mathbf{C}^{\prime}\right)$ consists of $\alpha c(\alpha \in \mathbf{R}-\{0\})$.

Let $h \in H_{\lambda}\left(\mathbf{C}^{\prime}\right)$. Since $h \in H_{i}\left(U^{\prime}\right)$ and $h \in H_{\lambda}\left(-U^{\prime}\right)$, Lemma 4 yields that $h \in H(\mathbf{C})$. Therefore $h$ takes the form

$$
h\left(r e^{i \Theta}\right)=\sum_{u=0}^{\infty} r^{n}\left(a_{n} \cos n \Theta+b_{n} \sin n \Theta\right) .
$$

Consider the functions

$$
\varrho_{n}(r)= \begin{cases}\left(r-\left(\eta_{n}+1\right)\right)\left(\left(\eta_{n}+1\right)^{2}-r\right), & r \in\left[\eta_{n}+1,\left(\eta_{n}+1\right)^{2}\right] ; \\ 0, & r \in[0, \infty)-\left[\eta_{n}+1,\left(\eta_{n}+1\right)^{2}\right],\end{cases}
$$

where $\left\{\eta_{n}\right\}$ is the sequence in no. 5. Assume that $a_{m}^{2}+b_{m}^{2} \neq 0$ for some $m>1$ and consider the functions

$$
\varphi_{n}\left(r e^{i \Theta}\right)=\varrho_{n}(r)\left(a_{m} \cos m \Theta+b_{m} \sin m \Theta\right) .
$$


By Theorem 1, $\left|\left(h, \varphi_{n}\right)_{\mu_{1}}\right|^{2}=\left|\left(h, \varphi_{n}\right)_{\lambda}\right|^{2} \leq K D_{\mathrm{C}^{\prime}}\left(\varphi_{n}\right)$ for a constant $K$ and for every $n=1,2, \ldots$ Observe that $\left(h, \varphi_{n}\right)_{\mu_{1}}=O\left(\eta_{n}^{2(m+1-\alpha)}\right)$ and $D_{\mathrm{C}^{\prime}}\left(\varphi_{n}\right)=O\left(\eta_{n}^{8}\right)$. Therefore $O\left(\eta_{n}^{2 m+2-2 \alpha}\right) \leq O\left(\eta_{n}^{4}\right)$ or $O\left(\eta_{n}^{m-(1+\alpha)}\right) \leq$ $O(1)$. Since $\eta_{n} \rightarrow \infty(n \rightarrow \infty)$, we must have $m \leq 1+\alpha<2$, a contradiction. Therefore $h=a+\alpha c+\beta s$. By Lemmas 1,2 and 3, $c \in H_{\lambda}\left(\mathbf{C}^{\prime}\right)$ and hence $a+\beta s \in H_{\lambda}\left(\mathbf{C}^{\prime}\right)$ unless $a+\beta s \equiv 0$. If $\beta \neq 0$, we can find a constant $\gamma>1$ such that $a+\beta s$ is of constant sign, say $>0$, in $\mathbf{Q}+\gamma$. Since $H_{\lambda}(\mathbf{Q}+\gamma)=H_{\mu_{1}+\mu_{2}}(\mathbf{Q}+\gamma)$,

$$
G_{\mathbf{Q}+\gamma}(a+\beta s, a+\beta s)_{i} \geq G_{\mathbf{Q}+\gamma}(a+\beta s, a+\beta s)_{\mu_{2}} .
$$

The right-hand side is seen to be $\infty$ by the proof of Lemma 2, a cor1tradiction. Hence $\beta=0$. If $a \neq 0$, say $a>0$, then $G_{\Omega}(a, a)_{\lambda} \rightarrow \infty$ as regular regions $\Omega$ exhaust $\mathbf{C}^{\prime}$ since $G_{\Omega} \rightarrow \infty\left(\Omega \rightarrow \mathbf{C}^{\prime}\right)$, again a contradiction. Therefore $a+\beta s \equiv 0$ and we deduce that $h=\alpha c$.

8. We compile the results in nos. $4-7$ and state:

Theorem 3. The Poisson equation $\Delta u=-\lambda h\left(h \in H\left(\mathbf{C}^{\prime}\right)\right)$ has a Dirichlet finite solution on $\mathbf{C}^{\prime}$ if and only if $h=\alpha c(\alpha \in \mathbf{R}-\{0\})$. Every Dirichlet finite solution $u$ of $\Delta u=-\lambda(\alpha c)$ on $\mathbf{C}^{\prime}$ is unbounded.

The first statement is nothing but Lemma 5 . To prove the second statement, we may assume $\alpha=1$. Let $\tilde{u}(z)=-u(j(z))$ where $j$ is the involution on $\hat{\mathbf{P}}^{\prime}$. Since $\lambda(j(z))=\lambda(z)$ and $c(j(z))=-c(z), \Delta \tilde{u}=-\lambda c$. Therefore $\quad \Delta(u-\tilde{u})=0$ and $u-\tilde{u} \in D\left(\mathbf{C}^{\prime}\right)$. By $\mathbf{C} \in O_{G} \subset O_{H D}$ (cf. e.g. Sario-Nakai [5]) $u-\tilde{u}=2 \alpha \in \mathbf{R}$, i.e. $u(z)+u(j(z))=2 \alpha$. In particular $u(z)=\alpha$ if $z \in \partial \mathbf{P}$. Let $\{\Omega\}$ be an exhaustion of $\mathbf{C}^{\prime}$ and $\omega=\Omega \cap \mathbf{P}^{\prime}$. As in the proof of Theorem 1,

$$
u=H_{u}^{(j}+(2 \pi)^{-1} \int_{\omega} G_{\omega}(\cdot, \zeta) c(\zeta) \lambda(\zeta) d \xi d \eta
$$

on $\omega$. Since $u=\alpha$ on $(\partial \omega) \cap(\partial \mathbf{P})$ and $\left\{H_{u}^{\omega}\right\}$ converges to a Dirichlet finite harmonic function $k$ on $\mathbf{P}^{\prime}, k \equiv \alpha$. By the Lebesgue-Fatou theorem,

$$
\begin{gathered}
u=\alpha+\frac{1}{2 \pi} \int_{\mathbf{P}^{\prime}} G_{\mathbf{P}}(\cdot, \zeta) c(\zeta) \lambda(\zeta) d \xi d \eta>\alpha+\frac{1}{2 \pi} \int_{\mathbf{P}^{\prime}} G_{\mathbf{P}}(\cdot, \zeta) c(\zeta) \mu_{5}(\zeta) d \xi d \eta \\
=\alpha-\frac{1}{2 \pi} \int_{\mathbf{P}^{\prime}} G_{\mathbf{P}}(\cdot, \zeta) \Delta \omega(\zeta) d \xi d \eta=\alpha+\omega .
\end{gathered}
$$

By (19), $\lim _{z \in \mathbf{P}^{\prime}, z \rightarrow 3} u(z)=\infty$, and $u$ is unbounded.

This completes the proof of Theorem 3 and hence of our main theorem. 


\section{References}

[1] Constantinescu, C. and Connea, A., Ideale Ränder Riemannscher Flächen Springer-Verlag, 1963.

[2] NaKat, M., Dirichlet finite solutions of $\Delta u=P u$, and classification of Riemann surfaces - Bull. Amer. Math. Soc. 77 (1971), $381-385$.

[3] -》- Dirichlet finite biharmonic functions on the plane with distorted metrics (to appear).

[4] Ozawa, M., Classification of Riemann surfaces, Kôdai Math. - Sem. Rep. 4 (1952), $63-76$.

[5] Sario, L. and NakaI, M., Classification Theory of Riemann Surfaces - SpringerVerlag, 1970.

[6] Virtanen, K. I., Über die Existenz von beschränkten harmonischen Funktionen auf offenen Riemannschen Flächen - Ann. Acad. Sci. Fenn. 75 (1950).

Santa Monica,

California, U.S.A. 